\title{
The red tail of carbon stars in the LMC: Models meet 2MASS and DENIS observations
}

\author{
P. Marigo ${ }^{1}$, L. Girardi ${ }^{2}$, and C. Chiosi ${ }^{1}$ \\ 1 Dipartimento di Astronomia, Università di Padova, Vicolo dell’Osservatorio 2, 35122 Padova, Italy \\ 2 Osservatorio Astronomico di Trieste, Via Tiepolo 11, 34131 Trieste, Italy
}

Received 9 December 2002 / Accepted 4 February 2003

\begin{abstract}
Carbon stars are known to exhibit systematically redder near-infrared colours with respect to M-type stars. In the near-infrared colour-magnitude diagrams provided by the 2MASS and DENIS surveys, the LMC C-type stars draw a striking "red tail", well separated from the sequences of O-rich giants. So far, this conspicuous feature has been absent from any set of available isochrones, even the few existing ones that include the TP-AGB evolution of low- and intermediate-mass stars. To investigate such issue we simulate the complete 2MASS $K_{\mathrm{s}}$ vs. $\left(J-K_{\mathrm{s}}\right)$ data towards the LMC by means of a population synthesis approach, that relies on extended libraries of published stellar evolutionary tracks, including the TP-AGB phase. The simulations provide quite a detailed description of the several vertical "fingers" and inclined sequences seen in 2MASS data, due to both galactic foreground and LMC O-rich stars. Instead, as mentioned, the red tail of C-stars sets a major difficulty: we find that TP-AGB models with solar-scaled molecular opacities, the usual assumption of existing AGB calculations, do not succeed in reproducing this feature. Our tests indicate that the main reason for this failure should not be ascribed to empirical $T_{\text {eff }}-(J-K)$ transformations for C-type stars. Instead, the discrepancy is simply removed by adopting new evolutionary models that account for the changes in molecular opacities as AGB stars get enriched in carbon via the third dredge-up (Marigo 2002). In fact, simulations that adopt these models are able to reproduce, for the first time, the red tail of C-stars in near-infrared CMDs. Finally, we point out that these simulations also provide useful indications about the efficiency of the third dredge-up process, and the pulsation modes of long-period variables.
\end{abstract}

Key words. stars: AGB and post-AGB - stars: evolution - stars: carbon - stars: fundamental parameters - stars: mass loss

\section{Introduction}

It has been already known for a long time that carbon (C-type) stars occupy a particular region of near-infrared colour-colour diagrams (e.g. Richer et al. 1979; Cohen et al. 1981; Frogel et al. 1990; Hughes \& Wood 1990). Specifically, C-type stars exhibit systematically redder colours than M-type stars, and particular colour-colour diagrams (e.g. $J-H$ vs. $H-K$ ) display such a sharp dichotomy between the two spectral classes that they are often used diagnostic tools to identify carbon star candidates from observed samples.

Recent wide-area near-infrared surveys like the Two Micron All Sky Survey (2MASS; Skrutskie et al. 1997) and the Deep Near-Infrared Southern Sky Survey (DENIS; Epchstein et al. 1997) have confirmed these findings. By providing photometric data for the complete AGB population of the Magellanic Clouds, they reveal the presence of a red plume of C-stars in a striking way (Cioni et al. 1999; Nikolaev \& Weinberg 2000). This feature can be appreciated in the $K_{\mathrm{s}}$ vs. $\left(J-K_{\mathrm{s}}\right)$ diagrams of Fig. 1: they show a marked almost-vertical sequence of red giants at $\left(J-K_{\mathrm{s}}\right) \sim 1.1$, from which an inclined branch departs at $K_{\mathrm{S}} \sim 11$ towards redder colours, extending up to $\left(J-K_{\mathrm{S}}\right) \sim 2.0$. This inclined branch is what we refer to as "the

Send offprint requests to: P. Marigo,

e-mail: marigo@pd.astro.it red tail". Nikolaev \& Weinberg (2000) and Cioni et al. (2001) demonstrate that the red tail is populated by non-obscured $\mathrm{C}$ stars, whereas the almost-vertical feature at $\left(J-K_{\mathrm{s}}\right) \sim 1.1$ is composed basically of O-rich giants (of spectral types from late-K to M). Moreover, the luminosities of red tail stars indicate that they belong to the thermally pulsing asymptotic giant branch (TP-AGB) phase, and hence they are expected to be the result of carbon surface enrichment by the recurrent third dredge-up process.

As for the modelling of this feature, the present-day situation is as follows. Most of the stellar isochrones for old and intermediate ages available in the literature do not include the TP-AGB phase, as they usually extend up to tip of the red giant branch (RGB) or the Early-AGB (e.g. Lejeune \& Schaerer 2001; Domínguez et al. 1999; Weiss \& Salaris 1999; Bergbusch \& VandenBerg 2001 and references therein). Two main reasons for this discrepancy are that i) fully modelling the AGB phase is indeed complex and time-consuming, and ii) most stellar models fail to explain the formation of carbon stars except for those at higher luminosities.

To our knowledge the only available isochrone sets that include TP-AGB stars are the Padova ones (Bertelli et al. 1994; Girardi et al. 2000; Salasnich et al. 2001; Marigo \& Girardi 2001). In these models the entire TP-AGB evolution is followed - with the aid of a synthetic approach to 

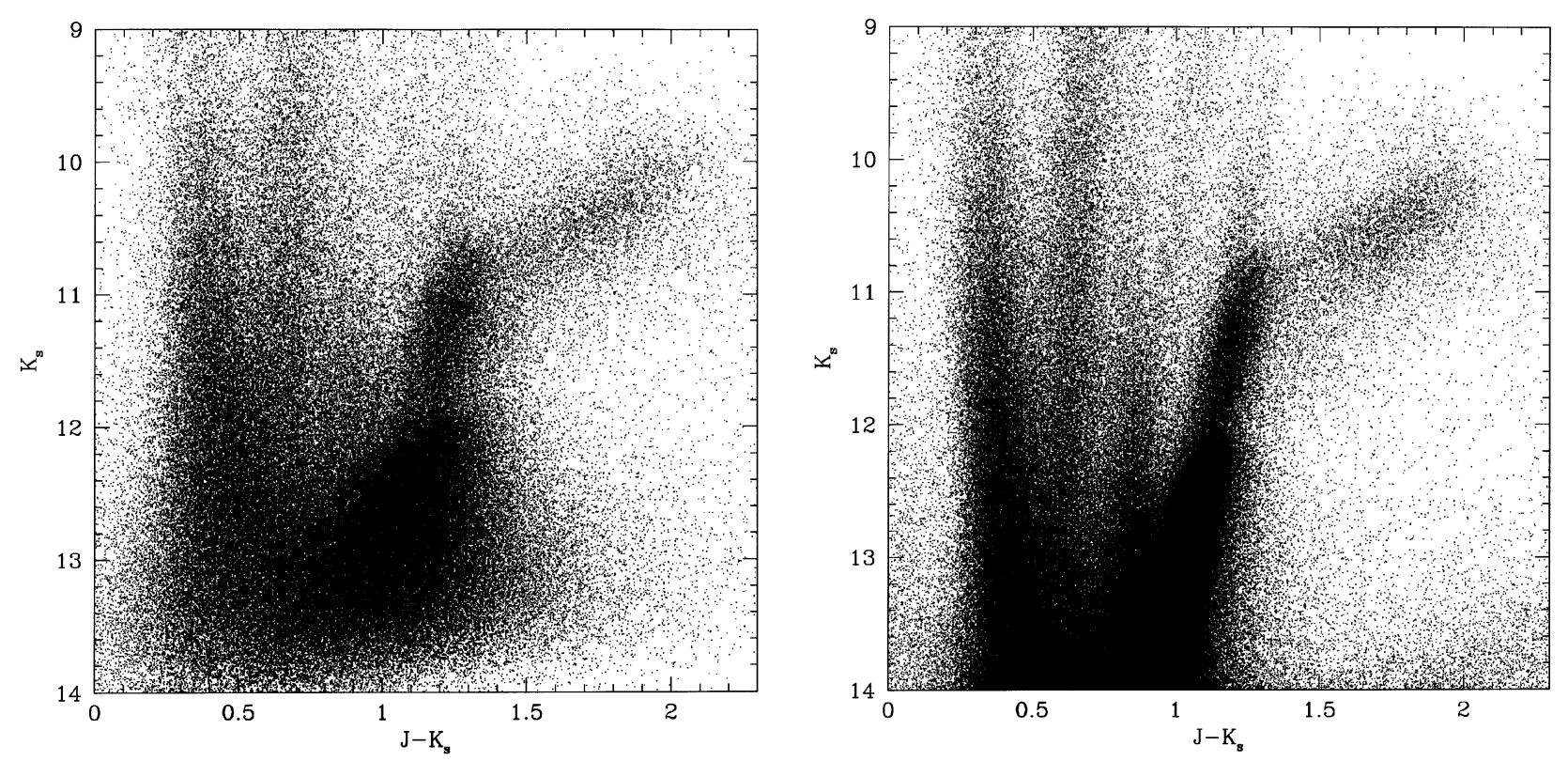

Fig. 1. Left panel: $K$ vs. $(J-K)$ diagram for the LMC region of sky $\left(101^{\circ} \leq \alpha \leq 61^{\circ}\right.$ and $\left.-77^{\circ} \leq \delta \leq-63^{\circ}\right)$. The data are taken from the 2MASS Second Incremental Data Release. Note the red tail of visible C-stars departing from the sequence of red giants at $(J-K) \sim 1.4$ and extending up to $(J-K) \sim 2$. Right panel: The same from DENIS Point Source Catalogue towards the LMC (Cioni et al. 2000).

various degrees of detail - up to ejection of the stellar envelope. However, all of them fail to predict the red tail of $\mathrm{C}$ stars, and in fact the reddest TP-AGB tracks hardly reach $(J-K)$ colours as large as 1.3. This is illustrated in Fig. 2. If in the older isochrone sets (Bertelli et al. 1994; Girardi et al. 2000) the basic problem was the lack of the C-star phase in the stellar models, the same does not affect the most recent models (Marigo \& Girardi 2001), that account for the formation of C-stars via the third dredge-up.

What is then the problem with the colours of theoretical C-star models? Why do they not reach $(J-K)$ as red as $\sim 2$ ? Since in Marigo \& Girardi (2001) isochrones the third dredgeup process is calibrated so that C-stars appear at the right luminosities (see Marigo et al. 1999 for details), the problem might be related either to (i) an improper transformation from $T_{\text {eff }}$ to the $(J-K)$ colour, which introduces errors of several tenths of a magnitude for C-type stars, and/or (ii) an improper modelling of the C-star radii and effective temperatures, with typical errors of some hundreds of degrees Kelvin.

In case of alternative (i), a simple revision of the $T_{\text {eff }}-$ colour relations, via e.g. the use of suitable relations for $\mathrm{M}$ - and $\mathrm{C}$ type stars, would solve the problem. In the case of option (ii), the implications would be much deeper, since the $T_{\text {eff }}$ values of Marigo et al. (1999) models are typical of most TP-AGB models in the literature.

Exploring both possibilities is the main scope of this paper, which is organised as follows. In Sect. 2 we start by recalling the main prescriptions in our synthetic TP-AGB models, that also account for the transition from O-rich (M-type) to C-rich (C-type) stars (Sect. 2.1). A brief summary of the present status of other AGB evolution models available in the literature is given in Sect. 2.2, putting particular emphasis on the role of molecular opacities.

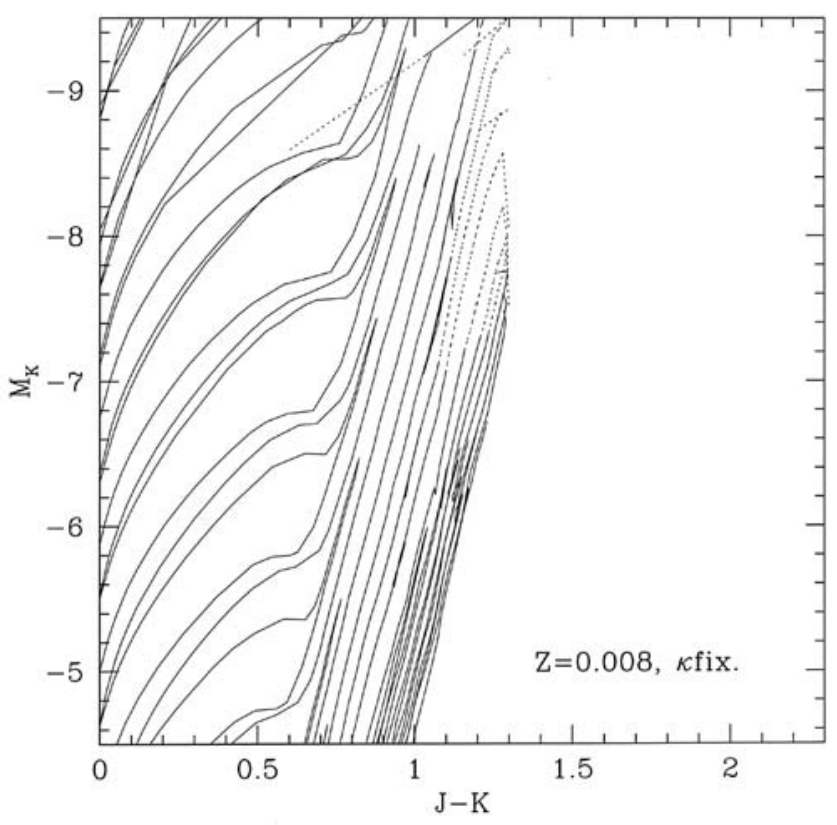

Fig. 2. A subset of theoretical isochrones described in Marigo \& Girardi (2001), and transformed to $J K$ photometry by means of Girardi et al. (2002) tables. TP-AGB models are computed with the $\kappa_{\mathrm{fix}}$ prescription. The metallicity is $Z=0.008$, and isochrones are presented at equally-spaced age intervals of $\Delta \log t=0.1$. The dotted lines mark the isochrone sections corresponding to $\mathrm{C}$-stars (with $\mathrm{C} / \mathrm{O}>1$ ). The plot limits correpond closely to those of Fig. 1 for a LMC distance modulus of 18.5 mag. In these and other models in literature, C stars do not form any red tail.

Section 3 describes our simulations of the 2MASS data in the $K$ vs. $(J-K)$ colour magnitude diagram (CMD). If several 2MASS features are reproduced in detail, the red tail of 
C-stars is missed by our initial models. Section 4 investigates the possible reasons for it. Finally, our main conclusions are summarised in Sect. 5.

\section{Models for C-stars}

\subsection{Our prescriptions: Old and new}

In this study the formation and evolution of C-stars is described with the aid of the synthetic TP-AGB model developed by Marigo et al. (1996, 1998, 1999), and Marigo (2002) to whom the reader is referred for all the details. For the sake of clarity let us briefly outline the main structure of the model and its basic prescriptions.

- The TP-AGB evolution - of a star of given initial mass and metallicity - is followed from the first thermal pulse up to the complete ejection of the envelope by stellar winds. This is performed by suitably coupling (i) analytic prescriptions (e.g. the core mass-luminosity relation, the core massinterpulse relation, the growth rate of the core mass during the quiescent stages), and (ii) a complete static envelope model to be integrated from the atmosphere down to the core.

- The initial conditions at the first thermal pulse are derived from sets of stellar tracks for low- and intermediate-mass stars by Girardi et al. (2000), which cover the previous evolutionary phases, i.e. from the zero-age main sequence up to the beginning of the TP-AGB phase. In this way consistency and homogeneity are guaranteed in the construction of the corresponding stellar isochrones (see Sect. 3.2).

- Third dredge-up episodes possibly take place at thermal pulses and eventually lead to the transition from $\mathrm{M}$ - to $\mathrm{C}$-class if the surface $\mathrm{C} / \mathrm{O}$ ratio increases above 1 . The third dredge-up is described as a function of two parameters, namely: the efficiency $\lambda$, and the minimum temperature $T_{\mathrm{b}}^{\mathrm{dred}}$ at the base of the convective envelope for dredgeup to take place. The previous calibration of these quantities (Marigo et al. 1999) on the observed luminosity functions of C-stars has yielded $\left(\lambda=0.50, \log T_{b}^{\text {dred }}=6.4\right)$ for the LMC and $\left(\lambda=0.65, \log T_{\mathrm{b}}^{\text {dred }}=6.4\right)$ for the SMC.

- Hot-bottom burning occurring in the most massive AGB stars $\left(M \gtrsim 4.5 M_{\odot}\right)$, is followed in detail with the aid of complete envelope integrations (Marigo et al. 1998; Marigo 1998). The possible break-down of the core massluminosity relation and the CNO-cycle nucleosynthesis (possibly preventing the formation of luminous C-stars) are both taken into account.

- Mass loss is included according to Vassiliadis \& Wood's (1993) formalism, based on observations of pulsating AGB stars. In our calculations we assume that pulsation occurs with periods $(P)$ corresponding to either the fundamental mode $\left(P_{0}\right)$, or the first overtone mode $\left(P_{1}\right)$.

- Of great importance for the analysis developed in this work are the opacity prescriptions to be used in the envelope model. At high temperatures $(T \geq 10000 \mathrm{~K})$ we adopt Iglesias \& Rogers (1993) for O- and C-rich mixtures. At low temperatures $(T<10000 \mathrm{~K})$ we use either the solarscaled opacity tables by Alexander \& Ferguson (1994; hereafter also $\kappa_{\mathrm{fix}}$ ), or the routine developed by Marigo (2002; hereafter also $\kappa_{\mathrm{var}}$ ) that predicts molecular opacities for any chemical composition of the gas. It should be remarked that before Marigo (2002), all TP-AGB tracks by Marigo et al. were calculated with $\kappa_{\text {fix }}$.

In summary, for the purpose of our present work we will make use of two groups of synthetic TP-AGB calculations, namely:

- old TP-AGB models with $\kappa_{\text {fix }}$ (Marigo et al. 1999);

- new TP-AGB models with $\kappa_{\text {var }}$ (Marigo 2002).

For the remaining prescriptions, we refer to the aforementioned model outline unless otherwise specified.

\subsection{The formation of $C$-stars in other models and the role of molecular opacities}

Before proceeding with our analysis it is worth recalling briefly the current status of AGB models available in the literature and the importance of a consistent choice in the use of molecular opacities.

First of all, the lack of efficient dredge-up in low-mass AGB models (say with $M \lesssim 2 M_{\odot}$ ) has been a difficulty shared by all full stellar evolution codes for a long time (the so-called "carbon star mystery", as referred to by Iben 1981). More recently this difficulty seems to be overcome by a few groups (e.g. Herwig et al. 1997; Straniero et al. 1997; Karakas et al. 2002), due to different numerical treatments in describing the convective boundaries.

However, even if some progress is achieved by full stellar evolution models in relation to the formation of low-mass C-stars, the distance between theory and observations is still large. Currently there is no complete set of evolutionary tracks - based on full stellar models - extending up to the end of the AGB phase for a sufficient coverage of stellar masses and metallicities. This prevents a systematic test of stellar models by comparison with observations, e.g. the luminosity functions of M- and C-stars, and simulations of CMDs including the most evolved AGB stars. The main reason is the heavy computational effort required to fully model the AGB phase. On the other hand, this limit can be totally removed if one allows for a certain loss of detail and opts for the more agile synthetic approach (see e.g. Groenewegen \& de Jong 1993; Marigo et al. 1996, 1998 for recent works).

Another heavy inadequacy of present stellar models (both full and synthetic) that has been recently highlighted by Marigo (2002) is the use of fixed solar-scaled molecular opacities in AGB models experiencing the third dredge-up.

In fact, this prescription (previously defined as $\kappa_{\text {fix }}$ case) is still the standard choice common to most full and synthetic evolutionary calculations ${ }^{1}$ of the AGB phase (e.g. Mouhcine \& Lançon 2002; Chieffi et al. 2001; Herwig 2000;

\footnotetext{
1 Actually, non-solar scaled molecular opacities were already taken into account in a simple way by Vassiliadis \& Wood (1993), but no significant difference emerged in comparison to models with solarscaled opacities, as the authors did not practically find carbon dredgeup in their AGB calculations.
} 


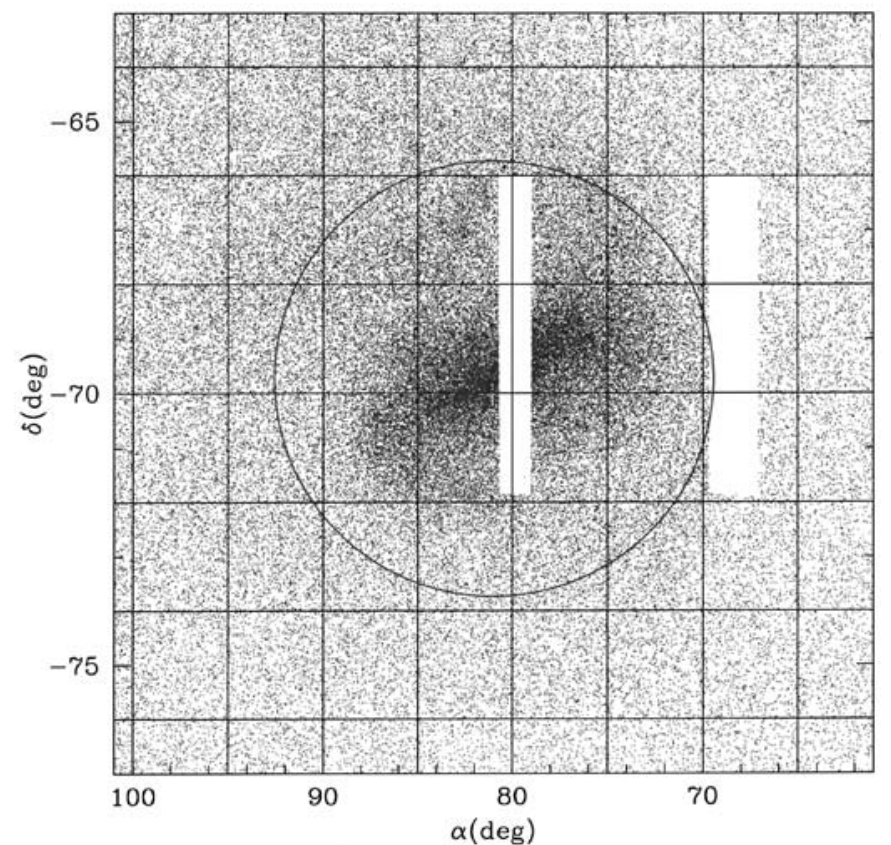

Fig. 3. Distribution of point sources from the 2MASS Second Incremental Data Release in the LMC region of the sky, projected on the $(\alpha, \delta)$ plane. To reduce confusion, we plot only the objects with $K_{\mathrm{s}}<12.5$. The circle delimits the area selected for our analysis.

Ventura et al. 1999; Wagenhuber \& Groenewegen 1998; Forestini \& Charbonnel 1997; Straniero et al. 1997).

Marigo (2002) has proved, instead, that properly coupling the molecular opacities to the actual surface chemical abundances $\left(\kappa_{\mathrm{var}}\right)$ brings along such important consequences that the standard evolutionary scenario for the AGB evolution may be significantly affected. The reader is referred to that work for a detailed analysis.

One major effect is that the surface enrichment in carbon that leads to $\mathrm{C} / \mathrm{O}>1$ - i.e. the formation of carbon stars causes a significant increase in opacities, which in turn is responsible for a sudden and marked cooling of the stellar tracks in the H-R diagram. The sizeable excursion towards lower $T_{\text {eff }}$, displayed by the $\kappa_{\mathrm{var}} \mathrm{C}$-star tracks, sets the first significant difference compared to $\kappa_{\text {fix }} \mathrm{C}$-star models that, instead, do not show any change in the slope of their evolutionary tracks while climbing the AGB at increasing luminosities.

A closer comparison between models with $\kappa_{\text {fix }}$ and $\kappa_{\mathrm{var}}$, and the impact of the new opacities prescription will be discussed in Sect. 4.2.

\section{The $K$ vs. $(J-K)$ diagram towards the LMC}

In this section, we aim to model the red tail of carbon stars in the LMC by using the TP-AGB models at our disposal. Our study is limited to a particular CMD - the $K$ vs. $(J-K)$ one - that reveals the red tail in all its prominence (see Fig. 1). Needless to say, the same feature is displayed in a variety of different CMDs involving near-infrared passbands, as can be seen e.g. in van der Marel \& Cioni (2001, their Fig. 2).

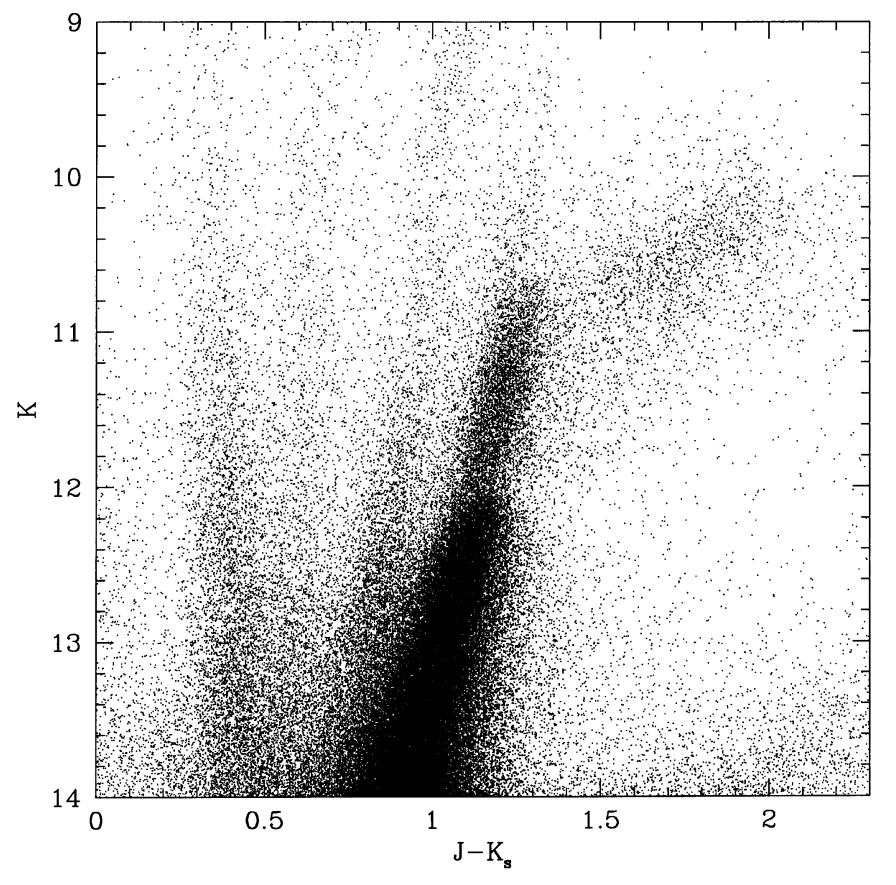

Fig. 4. 2MASS data for the innermost $4^{\circ}$ of the LMC. Note the red tail of visible C-stars extending up to $\left(J-K_{\mathrm{s}}\right) \sim 2$.

\subsection{The selected data}

In the following, we base our comparisons on 2MASS data towards the LMC, that present an extremely good photometric quality. More specifically, we use the point-source catalog of the 2MASS Second Incremental Data Release (see Cutri et al. 2002), which includes $10 \sigma$ detections as faint as $16.3,15.3$, 14.7 mag in $J, H$ and $K_{\mathrm{s}}$, respectively. As it can be seen in Fig. 3, these data wholly cover the LMC except for two limited strips of right ascension. In order to reduce the fore-/background of Galactic stars and distant galaxies, we select only the stars located closer than 4 degrees (projected) to the LMC bar optical center (at $\alpha=5^{\mathrm{h}} 24^{\mathrm{m}}, \delta=-69^{\circ} 44^{\prime}$, cf. Nikolaev \& Weinberg 2000). This region, also considering the empty strip, translates into to a total sky area of about 48.2 squared degrees. The corresponding CMD is shown in Fig. 4.

\subsection{Simulations}

The $K$ vs. ( $J-K$ ) diagram has been simulated by using a population synthesis code (Girardi et al., in preparation). In short, the code randomly generates stars following a given star formation rate (SFR), age-metallicity relation (AMR) and initial mass function (IMF). The stellar intrinsic properties (luminosity $L$, effective temperature $T_{\text {eff }}$, surface gravity $g$, etc.) are interpolated over a large grid of stellar evolutionary tracks, based on Bertelli et al. (1994) for massive stars, Girardi et al. (2000) for low- and intermediate-mass stars, and complemented with the TP-AGB tracks from either Marigo et al. (1999) with $\kappa_{\text {fix }}$, or new calculations with $\kappa_{\mathrm{var}}$ made on purpose (refer to Marigo 2002; see also Sect. 2).

The evolution along the TP-AGB phase is initially described in terms of the properties in the quiescent stages 

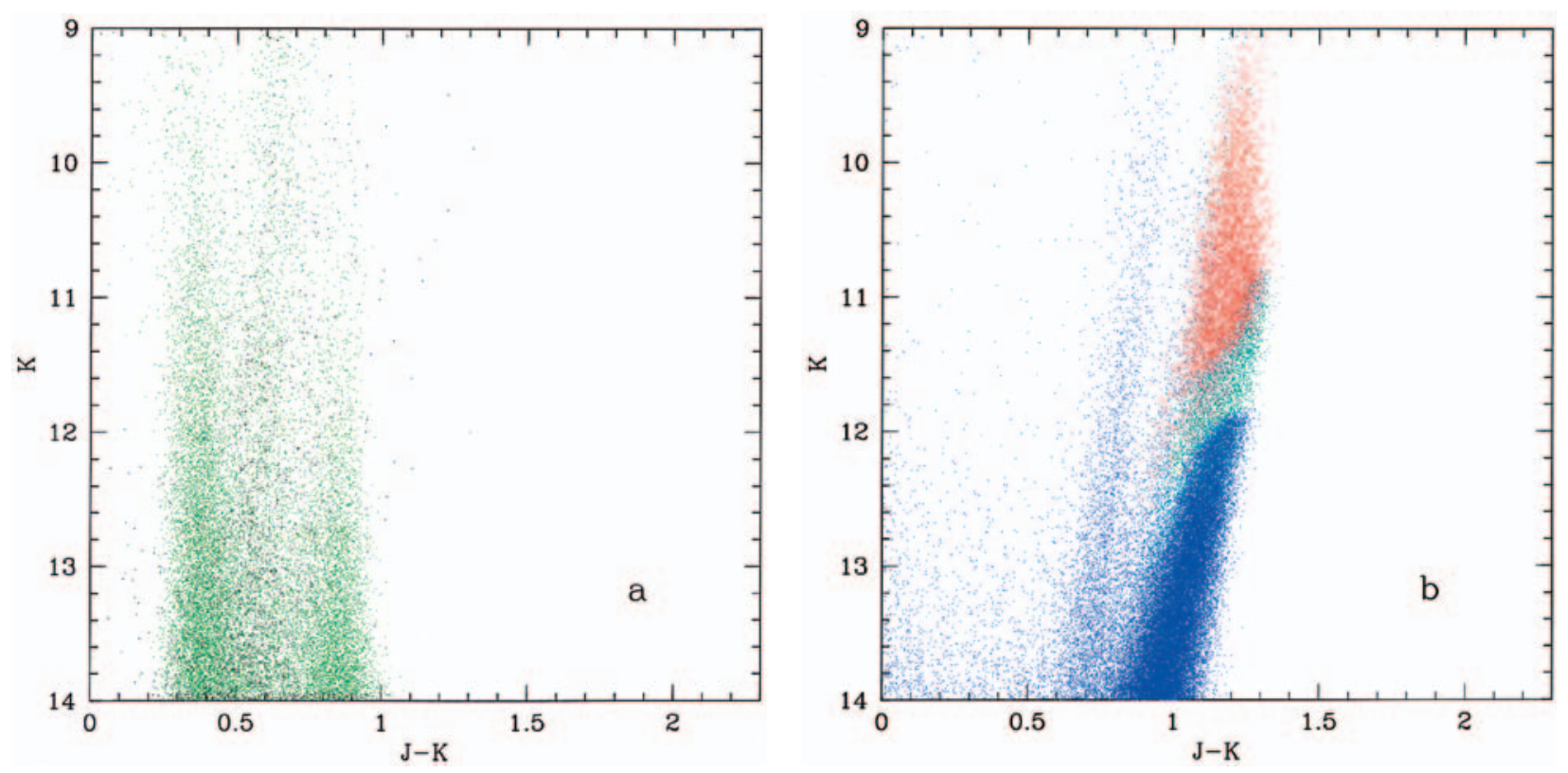

Fig. 5. Simulated CMDs for both the Galaxy foreground and the LMC population. Panel a) The Galaxy foreground stars. The coloured dots mark the stars belonging to the disk (green) and halo (black) populations. Panel b) The LMC population, marked in blue for all stars before the TP-AGB phase, and separated into O-rich (cyan) and C-rich stars (red) during the TP-AGB phase. Note that the C-star population distribute almost vertically over the red giant branch.

between thermal pulses. Actually, there are two additional factors that cause significant excursions in luminosity and effective temperature from their quiescent values, namely:

- occurrence of thermal pulses;

- instability against pulsation.

The former effect is included in our simulations. In practice, the luminosity variations driven by a thermal pulse (both the fast luminosity peak and long-lasting low-luminosity dip) are described according to the luminosity distributions over a pulse cycle as derived from Boothroyd \& Sackmann (1988), and assuming that the envelope mass is the main factor determining the shape of the luminosity dip. Once a star is randomly scattered in $\log L$ according to this distribution, the corresponding excursion in effective temperature is determined from a grid of envelope integrations performed in precedence. In this way, the intrinsic dispersion of TP-AGB stars in the HR diagram is realistically simulated with just a modest computational cost. We recall that this dispersion is important mainly for low-mass AGB stars, with a maximum displacement of $\approx-0.4$ in $\log L$ (or $\sim+1 \mathrm{mag}$ ), affecting up to 30 percent of their TP-AGB lifetimes.

As for the latter point related to stellar pulsation, it is worth premising the following. We recall that both 2MASS and DENIS provide single-epoch observations. Hence, the sampling of variable stars (e.g. Miras, SR variables) at a single and random phase of their variability cycle causes a further dispersion of data points in the observed CMD. This effect has not been simulated here for two reasons. First, we do not know exactly how $L$ and $T_{\text {eff }}$ vary during the pulsation cycle. Second, in any case the effect of this cyclical variation is easy to foresee: the bulk of TP-AGB stars will be scattered in the CMDs, to both higher and lower magnitudes and colours. The expected

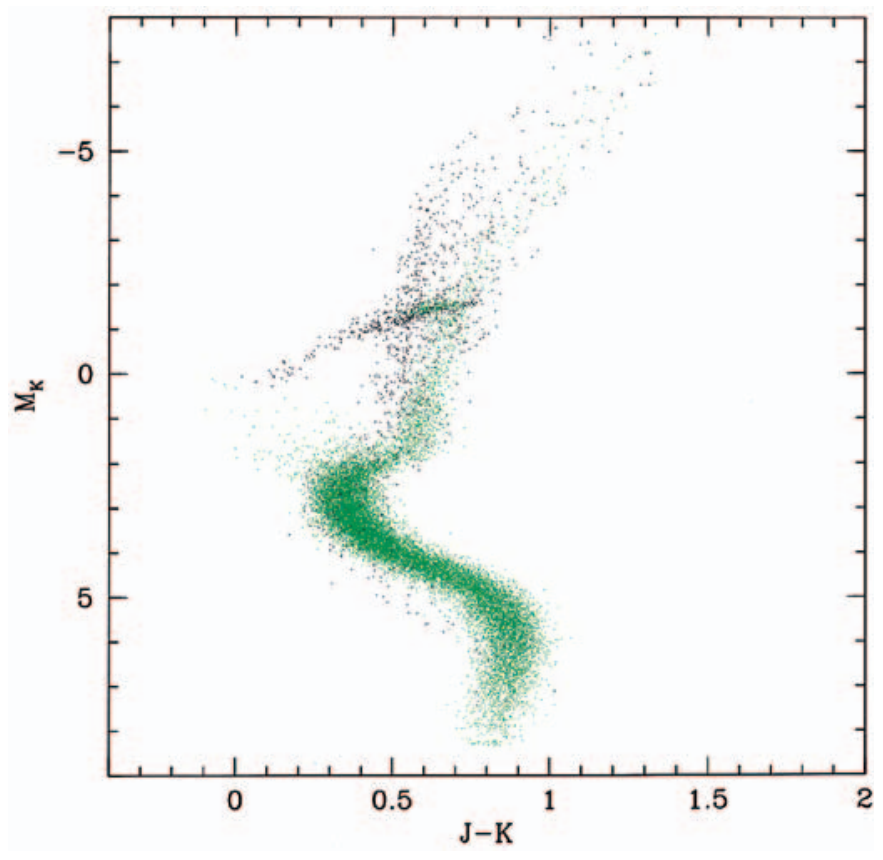

Fig. 6. The same data as in Fig. $5 \mathrm{a}$, but in the $M_{K}$ vs. $(J-K)$ plane.

result is a sort of general blurring of the TP-AGB sequences in the CMD. Mira and SR variables have pulsation amplitudes ranging from 0.4 to $1.0 \mathrm{mag}$ in the $I$-band (Hughes \& Wood 1990). Therefore, to take this effect into account the simulated TP-AGB sequences should be blurred by just some tenths of magnitude.

Once the stellar intrinsic properties are singled out, the photometry is generated by applying the extended tables of bolometric corrections from Girardi et al. (2002) for O-rich stars 
(with $\mathrm{C} / \mathrm{O}<1$ ), and empirical relations - to be discussed in Sect. 4.1 below - for C-type stars (with $\mathrm{C} / \mathrm{O}>1$ ).

Our simulations are computed in the $J H K$ filter system defined by Bessell \& Brett (1988). The expected differences from the 2MASS and DENIS systems - that use a " $K$-short" filter, $K_{\mathrm{S}}$ - are probably very low, of less than say $0.1 \mathrm{mag}$ in $(J-K)$. This is illustrated in the case of 2MASS by Cutri et al. (2002, Sect. II, 2d).

We simulate the photometric errors by means of a Monte Carlo approach, adopting the distribution of error values tabulated from 2MASS. We recall that $\sigma$ errors are lower than 0.03 mag for $K_{\mathrm{s}} \lesssim 13$.

Finally, the population synthesis code generates simulations for both the LMC galaxy and the Galactic foreground, by using different distributions of reddening and distances. Results are shown in Fig. 5, for the specific case of TP-AGB models computed with solar-scaled molecular opacities $\left(\kappa_{\text {fix }}\right)$, and discussed in the following.

\subsubsection{The Galactic foreground}

The field foreground stars have been included with the aid of a complete Galaxy model (Girardi et al., in preparation; Groenewegen et al. 2002). It consists of disk and halo components:

The disc is described by a double-exponential density law, with a radial scale length (in Galactocentric distance) of $2.8 \mathrm{pc}$, and a scale height $H$ that increases with stellar age $t$ as

$H=z_{0}\left(1+t / t_{0}\right)^{\alpha}$.

We adopt the parameters $z_{0}=95 \mathrm{pc}, t_{0}=0.5 \mathrm{Gyr}$ and $\alpha=1.66$, that well describe the disk components as derived by $\mathrm{Ng}$ et al. (1997). It is worth noticing that this prescription allows for a significant increase of $H$ at large ages, which is similar to assuming the presence of an old thick disk component. The distributions in mass, age and metallicity of the simulated disk stars are derived under the assumptions of: a constant star formation rate between $12 \mathrm{Gyr}$ ago and now; the empirical age-metallicity relation from Rocha-Pinto et al. (2000) with the addition of a dispersion of 0.2 dex in $[\mathrm{Fe} / \mathrm{H}]$ at any age; and the log-normal IMF from Chabrier (2001).

The halo is described by an oblate spheroidal (cf. Gilmore 1984) of axial ratio $q=0.65$, and a core radius of $2.8 \mathrm{Kpc}$. Halo stars are assumed to be old (from 12 to $13 \mathrm{Gyr}$ ), and to follow a Gaussian distribution of metallicities of mean $[\mathrm{Fe} / \mathrm{H}]=-1.6$ and standard deviation of 1.0 dex.

This set of prescriptions has been constrained by Groenewegen et al. (2002), by using a set of Deep Multicolor Survey (Osmer et al. 1998) and ESO Imaging Survey data (Prandoni et al. 1999). Interestingly, most of the calibration data were in visible pass-bands, whereas the model is now being applied to the interpretation of near-infrared data.

For the purpose of our study, we simulate a conic section of the Galaxy occupying the same sky area as in the selected 2MASS data. The field center has galactic coordinates $\ell=280^{\circ} .46, b=-32.89$. We recall that, for this particular line-of-sight, other Galactic components (spiral arms, the bulge) are of no relevance.
An important aspect is that in the modelling we can distinguish different kinds of stars and hence tackle the origin of the several observed features in the CMD of 2MASS.

Our field simulation is presented in (Fig. 5a). In this plot, we can identify three marked vertical sequences of foreground disk stars (green dots), located at $(J-K)$ colours of about 0.35 , 0.65 , and 0.9 . Their origin is easily understood by looking at the corresponding plot in absolute magnitude (Fig. 6): The sequence to the left (at $(J-K) \simeq 0.35$ ) is defined mainly by the old disk turn-off, with typical masses of $M \simeq 0.9 M_{\odot}$; the central one (at $(J-K) \simeq 0.65)$ hosts fainter RGB and red clump stars; and the right one (at $(J-K) \simeq 0.9$ ) corresponds to lowmass dwarfs with $M \lesssim 0.6 M_{\odot}$. The vertical development of these strips in the CMD (Fig. 5a) simply reflects the large range of distances involved.

We notice that disk stars younger than $\sim 4$ Gyr $(M \gtrsim$ $1.3 M_{\odot}$ ) do not populate significantly this diagram, since they are found preferentially at small scale heights and hence at lower galactic latitudes and brighter apparent magnitudes (i.e. $K \lesssim 10$ ). The contribution of halo stars (black points) is also of minor importance, accounting for just a handful of points in the synthetic CMD, mostly at fainter apparent magnitudes (i.e. $K \gtrsim 13$ ), due to their large mean distances.

Finally, an important point is that field stars are not expected to reach colours redder than $(J-K) \simeq 1.0$, so that they hardly contaminate the CMD features produced by the LMC population of AGB stars. Their intepretation is our next goal.

\subsubsection{The LMC population}

The LMC population has been initially described by means of a very simplified model, under the assumptions of i) a fixed distance of $52.3 \mathrm{Kpc}$ (Girardi \& Salaris 2001), ii) constant SFR from 15 Gyr ago to now, and iii) a constant metallicity, $Z=0.008$, at any age. The latter choice is made for the sake of simplicity, since it requires the use of a single set of evolutionary tracks, hence relieving us, for the moment, from the effort to compute complete sets of TP-AGB models for several metallicities ${ }^{2}$. Anyway, the quality of our results should not depend much on these assumptions ${ }^{3}$.

In order to get a number of LMC giants comparable to the observed sample (Fig. 4), we have to simulate a total star formation of $5 \times 10^{8} M_{\odot}$ during the entire LMC history. Of course, this is just a rough estimate of the mass consumed to form stars, its value depending on the adopted assumptions about SFR (constant), IMF (from Kroupa 2001), and size of the simulated region (i.e. within the innermost $4^{\circ}$ of the LMC field). Anyway, it is interesting to notice that this number is just an order of magnitude lower than the total LMC mass (including dark matter; see van der Marel et al. 2002), and comparable to the present LMC H i mass (Staveley-Smith et al. 2003).

\footnotetext{
${ }^{2}$ Extended sets of TP-AGB tracks and isochrones for a wide range of metallicities are being computed and are soon to become available.

${ }^{3}$ In one of our test cases, we used TP-AGB tracks interpolated within an extended grid of 5 different metallicities, together with a more realistic AMR (from Pagel \& Tautvaisiene 1998). No significant difference was noticed in the resulting CMDs.
} 
The simulated LMC population draws a few almost vertical features in the CMD (Fig. 5b), mainly populated by: young and intermediate-age main-sequence stars (with $(J-K)<0.5$ ), intermediate-mass stars in the stage of core-He burning (the long diagonal sequence at $(J-K) \simeq 0.8)$, intermediate-age and old RGB stars of larger luminosities (the prominent sequence at $(J-K) \simeq 1$ and with $K>12)$, and intermediate-mass stars during the early-AGB phase (the weaker sequence departing slightly from the left of the RGB-tip, with the same inclination as the RGB). All these features (marked with blue dots) correspond to O-rich stars in evolutionary stages previous to the TP-AGB.

The O-rich TP-AGB stars (marked with cyan dots) trace two well-defined sequences: the first consists of intermediatemass stars that somewhat extend the plume of early-AGB stars towards higher luminosities; the second defines a clear strip located directly above the RGB-tip, corresponding to low-mass stars. Finally, The C-rich TP-AGB stars (marked with red dots) distribute above and to the right of these latter sequences.

Combining both panels of Fig. 5, it turns out that our initial simulations explain quite well the several "fingers" displayed by the 2MASS data of Fig. 4, but for one major discrepancy: the location of C-stars, predicted by these particular models, simply align above the sequence of LMC O-rich giants, failing to form the red tail. As for the other CMD features, the agreement with data is indeed quite good, and our interpretations substantially agree with those advanced by Nikolaev \& Weinberg (2000).

\section{Probing the red tail of C-stars}

In the following we will focus on the region of our simulated diagrams populated by TP-AGB stars, with the aim of casting light on the main cause that produces the observed red tail of $\mathrm{C}$-stars in near-infrared colors. We investigate two possible factors separately, namely: the colour- $T_{\text {eff }}$ relations (derived from empirical calibrations), and the evolution in effective temperatures (derived from stellar models).

\subsection{Testing different colour- $T_{\text {eff }}$ relations}

Let us start by analysing the effect on our simulations of different $(J-K)-T_{\text {eff }}$ transformations. It is important to remark that all computations discussed in this section are carried out by adopting TP-AGB tracks for solar-scaled $\kappa_{\text {fix }}$ opacities. Results are shown in Fig. 7.

As a first step, in Sect. 4.1.1 we consider the sharp dichotomy between colour- $T_{\text {eff }}$ relations derived from observed M-type stars (e.g. Fluks et al. 1994) and C-stars (e.g. Bergeat et al. 2001). Such differences essentially reflect the abrupt change in the dominant molecular species - producing the line blanketing - when passing from an O-rich spectrum (e.g. TiO, VO, $\mathrm{H}_{2} \mathrm{O}$ ) to a C-rich one (e.g. $\mathrm{C}_{2}, \mathrm{CN}, \mathrm{SiC}$ )

Then, within the class of C-stars, in Sect. 4.1.2 we attempt to account, in a simple way, for the possible composition dependence (related to the extent of carbon enrichment) of colour- $T_{\text {eff }}$ relations.

\subsubsection{A $T_{\text {eff }}-(J-K)$ relation valid for Galactic C-stars}

Figure 7a shows our initial simulation: The TP-AGB models are calculated with $\kappa$-fix molecular opacities, and the conversion from $T_{\text {eff }}$ to $(J-K)$ is based on Fluks et al. (1994) empirical spectra and temperature scale for nearby $M$ giants (see Girardi et al. 2002 for details). This means that a relation valid for O-rich stars has been applied also to C-stars. The discrepancy with observed data is obvious: C-stars just align above the sequence of O-rich stars, and do not develop any hint of red tail.

As a first attempt to include proper relations valid for $\mathrm{C}$ stars, we apply the Bergeat et al. (2001) formulas, that give $K$ band bolometric corrections and $(J-K)$ as a function of $T_{\text {eff }}$. These fitting relations are derived from a sample of Galactic $\mathrm{C}$ stars. In this context we would like to note that the Bergeat et al. (2001) relationship is essentially the same as that of Bessell et al. (1981). The resulting simulations are shown in Fig. 7b. With respect to the previous case, the sequence of C-stars is shifted to the blue, and not to the red as required to explain the data.

In this and other plots we show next, we have also adopted the empirical $K$-band bolometric corrections for C stars presented by Frogel et al. (1990). They differ little (by $\sim 0.1 \mathrm{mag}$ ) from those independently derived by Bergeat et al. (2001), and yield closely similar simulated CMDs.

\subsubsection{The effect of molecular blanketing for C-stars}

Molecular blanketing is responsible for the distinction between $\mathrm{M}$ - and C-spectral features and also affects the infrared colours of C-stars themselves (e.g. Cohen et al. 1981; Loidl et al. 2001). In this latter case, the extent of molecular blanketing is essentially related to the amount of carbon available to form molecules other than CO.

In their study of Galactic and LMC C-stars, Cohen et al. (1981) pointed out that the observed mean $(J-K)$ colour increases steadily as the carbon abundance class - defined by the $\mathrm{C}_{2}$ band strength - increases (from say C,2 to C,5). Moreover, the authors showed that, for a given effective temperature, redder $J H K$ colours are predicted by model atmospheres with larger $\mathrm{C}_{2}$ and $\mathrm{CN}$ enhancements.

In our work we attempt to account, in a simple way, for the effect of molecules on the infrared colours of C-stars, by introducing a $\mathrm{C} / \mathrm{O}$-dependence into the empirical $(J-K)-T_{\text {eff }}$ relation by Bergeat et al. (2001). The C/O data for Galactic Cstars are taken from Lambert et al. (1986). Figure 8 displays the observed trends of the $(J-K)$ colour as a function of $T_{\text {eff }}$ and $\mathrm{C} / \mathrm{O}$ ratio. The linear bi-parametric fit relation to the data is:

$(J-K)=17.32-4.56 \log T_{\text {eff }}+0.052 \mathrm{C} / \mathrm{O}$.

We see that the $(J-K)-T_{\text {eff }}$ relation (top-left panel of Fig. 8) is quite narrow for galactic $\mathrm{C}$-stars, and it is well reproduced by our fitting formula for $\mathrm{C} / \mathrm{O}=1.1$, in agreement with the observational finding that most Galactic carbon stars have low C/O ratios, not much larger than one (Lambert et al. 1986). Moreover, despite some observed scatter and the small number of data points, we also note that there is a positive correlation 

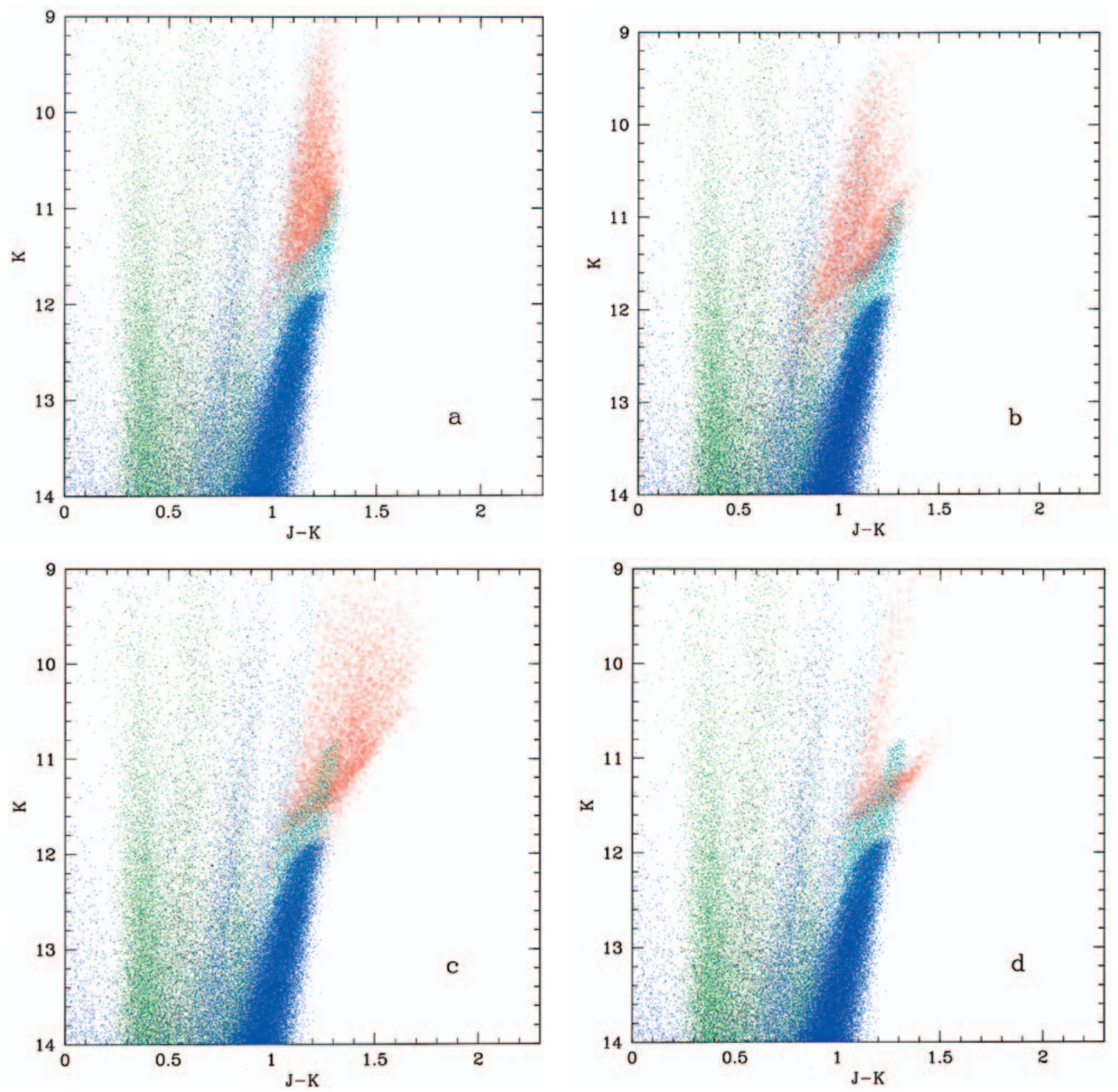

Fig. 7. Simulated CMDs based on TP-AGB tracks with $\kappa_{\text {fix }}$ prescription for molecular opacities. The simulations includes both the Galaxy foreground and the LMC population. The coloured dots mark several kinds of stars: foreground stars in the Galaxy disk (green) and halo (black); LMC giants before the TP-AGB phase (blue), and separated into O-rich (cyan) and C-rich stars (red) during the TP-AGB phase. Note that the C-star population distribute almost vertically over the red giant branch. Different $(J-K)$ vs. $T_{\text {eff }}$ transformations are tested in the simulations of the C stars: Panel a) The same as for M stars (based on Fluks et al. 1994); Panel b) Bergeat et al. (2001) one; Panel c) A fitting relation involving $\mathrm{C} / \mathrm{O}$ (Eq. (1)). A modest inclination appears in the C-star sequence. Panel d) The same as in panel c), but plotting only the data with a maximum $\mathrm{C} / \mathrm{O}$ value of 2.0.

between the $(J-K)$ colour and the $\mathrm{C} / \mathrm{O}$ ratio (bottom-left panel of Fig. 8). This trend is included in our fitting relation as it predicts a systematic shift towards redder $(J-K)$ colours for e.g. $\mathrm{C} / \mathrm{O}=3.0$, compared to the case $\mathrm{C} / \mathrm{O}=1.1$ (top left panel).

Overall, Eq. (1) fits the data to better than $0.1 \mathrm{mag}$ in $(J-K)$, as indicated by the residuals in the right panels of Fig. 8.

Actually, in a recent study Matsuura et al. (2002) have pointed out that the molecular features in the spectra of LMC $\mathrm{C}$-stars are consistent with $\mathrm{C} / \mathrm{O}$ ratios larger than those of Galactic C-stars. This empirical finding completely supports the results of our TP-AGB calculations, as shown in Fig. 9.
The same figure indicates that, in order to model the LMC population, we will have to apply Eq. (1) to $\mathrm{C} / \mathrm{O}$ values as high as 4 , that is beyond the maximum observed values of Galactic C-stars.

Thus, by using Eq. (1) in our simulations, we get the synthetic CMD of Fig. 7c. As it can be noticed, the dependence of $(J-K)$ on the $\mathrm{C} / \mathrm{O}$ ratio now causes the sequence of C-stars to be significantly redder, reaching $(J-K)=1.7$. However, the C-stars are still largely distributed among and above the O-rich giants, and no clear red tail is drawn. 

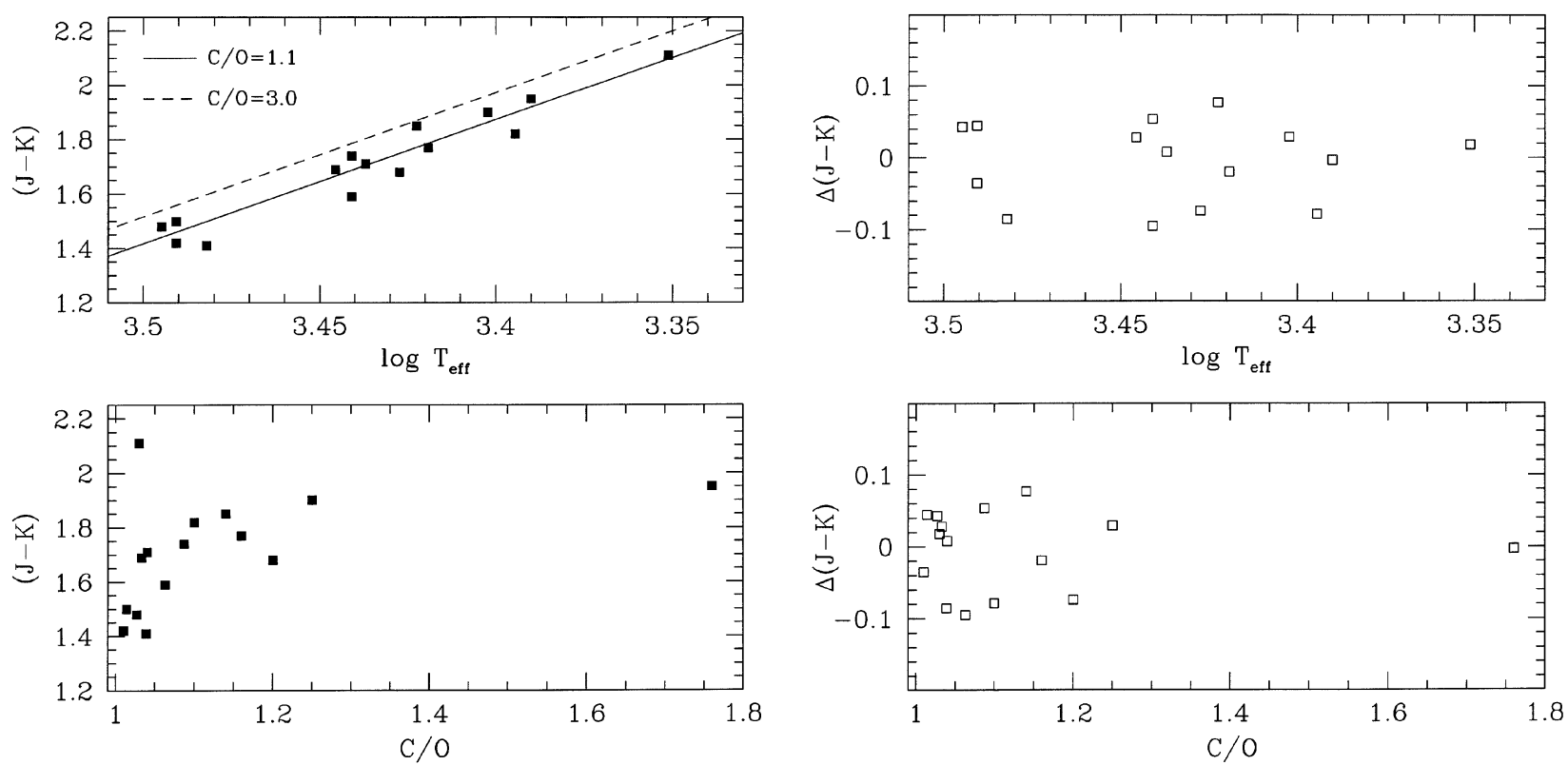

Fig. 8. Left panels: $(J-K)$ vs. $T_{\text {eff }}$ and $(J-K)$ vs. C/O relations for galactic C-stars. Observed data (filled squares) are from Bergeat et al. (2001, objects common to their tables 4 and 13). In the upper panel, lines show the predictions of the fit relation given by Eq. (1), assuming a constant C/O ratio, equal to 1.1 (solid) and 3.0 (dashed). Right panels: The residuals of fitting Eq. (1) to the data, plotted as a function of both $T_{\text {eff }}$ and $\mathrm{C} / \mathrm{O}$. The data is well reproduced by this relation to within $0.1 \mathrm{mag}$.

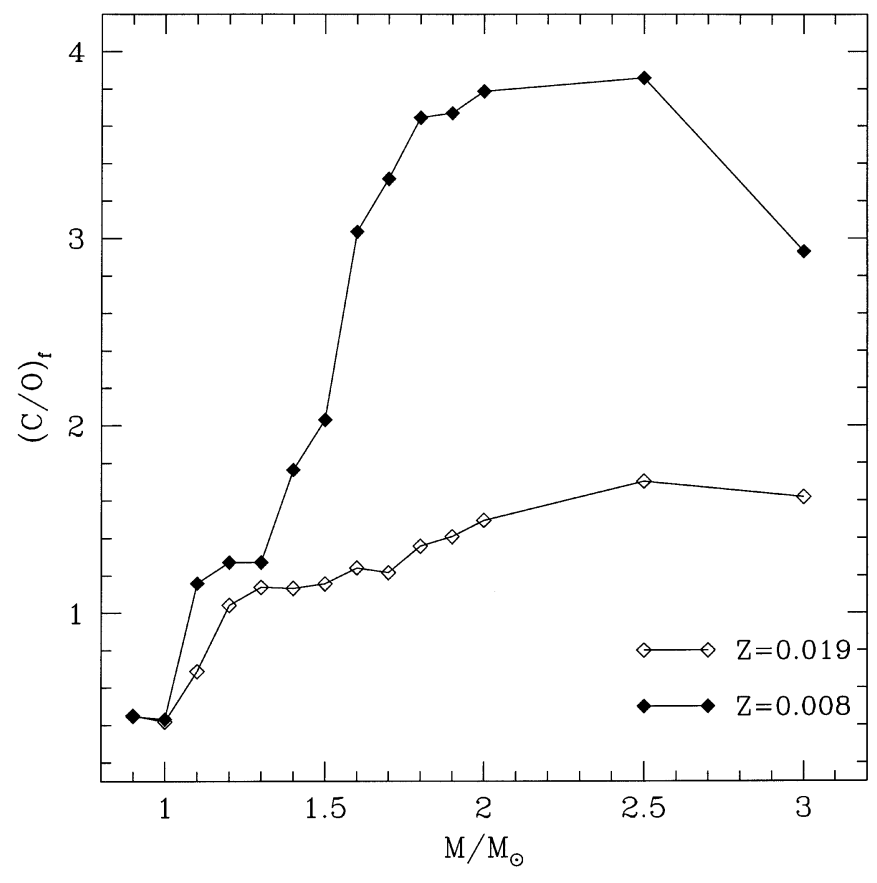

Fig. 9. Final $\mathrm{C} / \mathrm{O}$ ratio (at the end of the TP-AGB phase) as a function of the stellar mass (at the onset of the TP-AGB phase), predicted by synthetic models with variable molecular opacities $\kappa_{\mathrm{var}}$, and parameter set $\left(\lambda=0.50, \log T_{\mathrm{b}}^{\text {dred }}=6.4\right)$. Results for solar metallicity $(Z=$ $0.019)$ are compared to those for LMC composition $(Z=0.008)$.

If we limit consideration to models with $\mathrm{C} / \mathrm{O}<2$, i.e. plotting only those stars for which Eq. (1) is strictly valid, we get Fig. 7d. In this case, we can notice that the low-C/O C-stars contaminate significantly the $\mathrm{CMD}$ region corresponding to $\mathrm{O}$ rich AGB stars. On the contrary, such a high fraction of C-stars mixed up with M-stars is not present in observed samples. It is clear that this discrepant feature would remain for whatever hypothetical $(J-K)-T_{\text {eff }}-\mathrm{C} / \mathrm{O}$ that could push somehow the models to reach $(J-K)$ colours as high as 2.0.

In conclusion, our test calculations seem to indicate that the principal cause of the failure of models with solar-scaled molecular opacities in reproducing the red tail of carbon stars should not be ascribed to the $(J-K)-T_{\text {eff }}$ relation.

\subsection{Testing different TP-AGB tracks}

If we assume that Eq. (1) reasonably well describes the $(J-K)$ colours of C-stars, as supported by the low fitting residuals (of $\lesssim 0.1 \mathrm{mag}$, see the right panels of Fig. 8), we are left with just one alternative: redder $(J-K)$ colours could be attained only by assigning lower $T_{\text {eff }}$ to C-star models. Moreover, in order to locate the $\mathrm{C}$-star models onto a different branch from that of the O-rich models, a net $T_{\text {eff }}$ segregation between the two classes turns out to be necessary. These requirements are indeed fulfilled by Marigo (2002) TP-AGB models with variable molecular opacities.

Figure 10 illustrates a set of $Z=0.008$ isochrones based on Marigo's (2002) models. Similar isochrone sets have been computed for a large variety of dredge-up parameters and metallicities, and will be extensively described in a future paper. The conversion to $(J-K)$ colours for $\mathrm{C}$-star models is now based on Eq. (1). It is also interesting to notice that in this case we could not even have applied the same colour transformations derived for M-stars, since the effective temperatures of C-star models with $\kappa_{\mathrm{var}}$ are already much cooler than the validity lower-limits of such relations. 


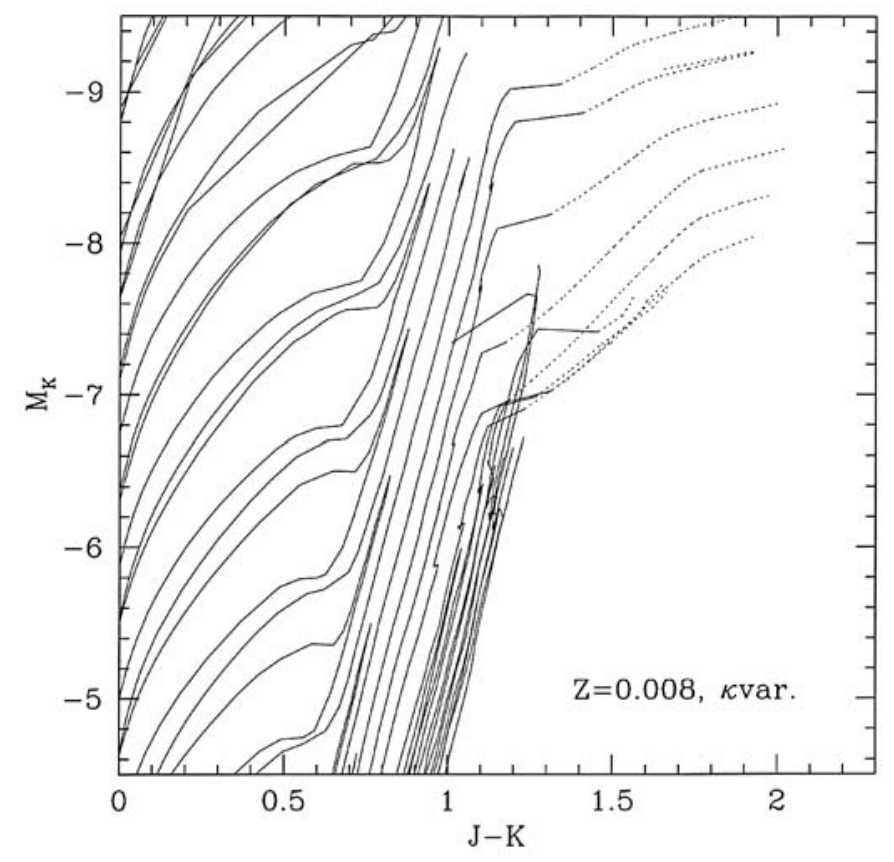

Fig. 10. $Z=0.008$ theoretical isochrones for TP-AGB models computed with variable molecular opacities. The dredge-up parameters are the same as in the models of Fig. 2. The dotted lines mark the isochrone sections corresponding to C-stars. The conversion to the $(J-K)$ photometry is now based on Eq. (1).

\subsubsection{The sole effect of variable molecular opacities}

The effect of TP-AGB models with variable molecular opacities is illustrated in the simulations of Fig. 11. TP-AGB models are computed with different assumptions (see also Sect. 2) regarding the onset of the third dredge-up - controlled by the parameter $T_{\mathrm{b}}^{\text {dred }}-$, and the onset of the super-wind regime - depending on the pulsation mode, either fundamental $\left(P=P_{0}\right)$, or first overtone $\left(P=P_{1}\right)$. In all cases, the sequence of C-stars clearly departs from the branch of O-rich stars.

In these $\kappa_{\mathrm{var}}$ models the excursion towards redder $(J-$ $K)$ colours is mostly caused by the displacement towards cooler $T_{\text {eff }}$. Instead, the increase of the $\mathrm{C} / \mathrm{O}$ ratio during the TP-AGB evolution should play a smaller role. In fact, considering that the maximum $\mathrm{C} / \mathrm{O}$ ratio reached by these models is of about 4 (Fig. 9), the C/O-dependence in the colour transformation of Eq. (1) accounts for at most 0.2 mag of the total colour excursion at the end of the $\mathrm{C}$-star evolution, the mean value being of about $0.1 \mathrm{mag}$.

In Fig. 11a, we adopt the same dredge-up parameters as calibrated in Marigo et al. (1999), i.e. $\log T_{\mathrm{b}}^{\text {dred }}=6.4$ and $\lambda=0.5$. The simulated C-star red tail resembles very much the observed one, but for two aspects.

First, the red tail shows up at a too low luminosity. It starts at $K=11.6$ when $(J-K)=1.2$, whereas the observed tail is at least $0.6 \mathrm{mag}$ brighter. This problem can be easily solved with a re-calibration of the dredge-up parameters. To give an example, Fig. $11 \mathrm{~b}$ shows a simulation that makes use of TP-AGB models recomputed with $\log T_{\mathrm{b}}^{\text {dred }}=6.45$. This parameter choice has the effect to delay the onset of the third dredge-up to higher stellar core masses and hence higher luminosities. As we can see, now the red tail starts at $K=11.0$ when $(J-K)=1.2$, which is about the right luminosity level to fit the data.

It is worth remarking that in both simulations presented in the upper panels ( $a$ and $b$ ) of Fig. 11, we have assumed that all TP-AGB stars are fundamental-mode pulsators, meaning that the Vassiliadis \& Wood's (1993) mass-loss formalism is applied with $\dot{M}(P)=\dot{M}\left(P_{0}\right)$. This assumption introduces the second problem of the models, which is discussed below.

\subsubsection{Extending the red tail with first-overtone pulsators}

The mentioned second theoretical difficulty resides in the extension of the red tail, which in both simulations (Figs. 11a, b) clearly reaches $(J-K)=1.8$, but hardly $(J-K)=2.0$ as in the observations. Although this point could be considered as a minor discrepancy (at least compared with the $\kappa_{\text {fix }}$ cases illustrated in Fig. 7), it deserves some attention. There are at least two possible ways out. The first is that we have not considered the effect of circumstellar reddening on the colours of C-stars. This should be ascribed to thick circumstellar material ejected by the coolest $\mathrm{C}$-stars during an extreme mass-loss phase. In fact, a few LMC C-stars are known to extend up to $(J-K) \sim 6$ (Cioni et al. 1999; Nikolaev \& Weinberg 2000); they are among the so-called "obscured" or "dust-enshrouded" C-stars identified with IRAS sources (see van Loon 1999), which are found to undergo heavy mass-loss. The question, however, is if this sort of self-reddening is already effective in red tail C-stars with say $(J-K)_{0} \lesssim 1.8$, being able to shift their colours up to $(J-K)=2.0$. This point has to be investigated with the aid of more detailed modelling.

The second explanation stands on the assumption that the dominant pulsation mode of AGB stars is the first overtone (with period $P=P_{1}$ ). This would allow us to get simulated colours further increased by $0.2 \mathrm{mag}$, while keeping the same $T_{\text {eff }}$ vs. $(J-K)$ relation. This is illustrated in the bottom panels of Fig. 11. For these simulations we use TP-AGB models computed with the same dredge-up parameters as in the upper panels, but assuming that Mira and SR variables are first overtone pulsators. Under this hypothesis, variable AGB stars should have shorter pulsation periods for given stellar $L$ and $T_{\text {eff }}$, compared to the fundamental-mode counterparts.

As a consequence, by virtue of the empirical positive correlation between the mass-loss rates and the periods of Mira stars, we expect that for the shorter first overtone periods $\left(P_{0} / P_{1} \sim\right.$ 2.2) the onset of the super-wind regime is postponed, thus implying that the end of the AGB evolution is delayed. We quantify this effect by adopting the Vassiliadis \& Wood's (1993) formalism with $\dot{M}(P)=\dot{M}\left(P_{1}\right)$ (Figs. 11c, d). In this way we find that $\mathrm{C}$-star models are allowed to reach slightly higher $L$ and lower $T_{\text {eff }}$, and their lifetimes increase accordingly. This latter effect is also relevant if we consider that longer lifetimes are needed to better reproduce the observed C-star counts in LMC clusters (Girardi \& Marigo 2003, and work in preparation).

In Fig. 11c, we can notice that now the red tail actually extends up to $(J-K)=2.0$. However, it still appears at too faint luminosities, due to the low value assumed for $T_{\mathrm{b}}^{\text {dred }}$ (see 

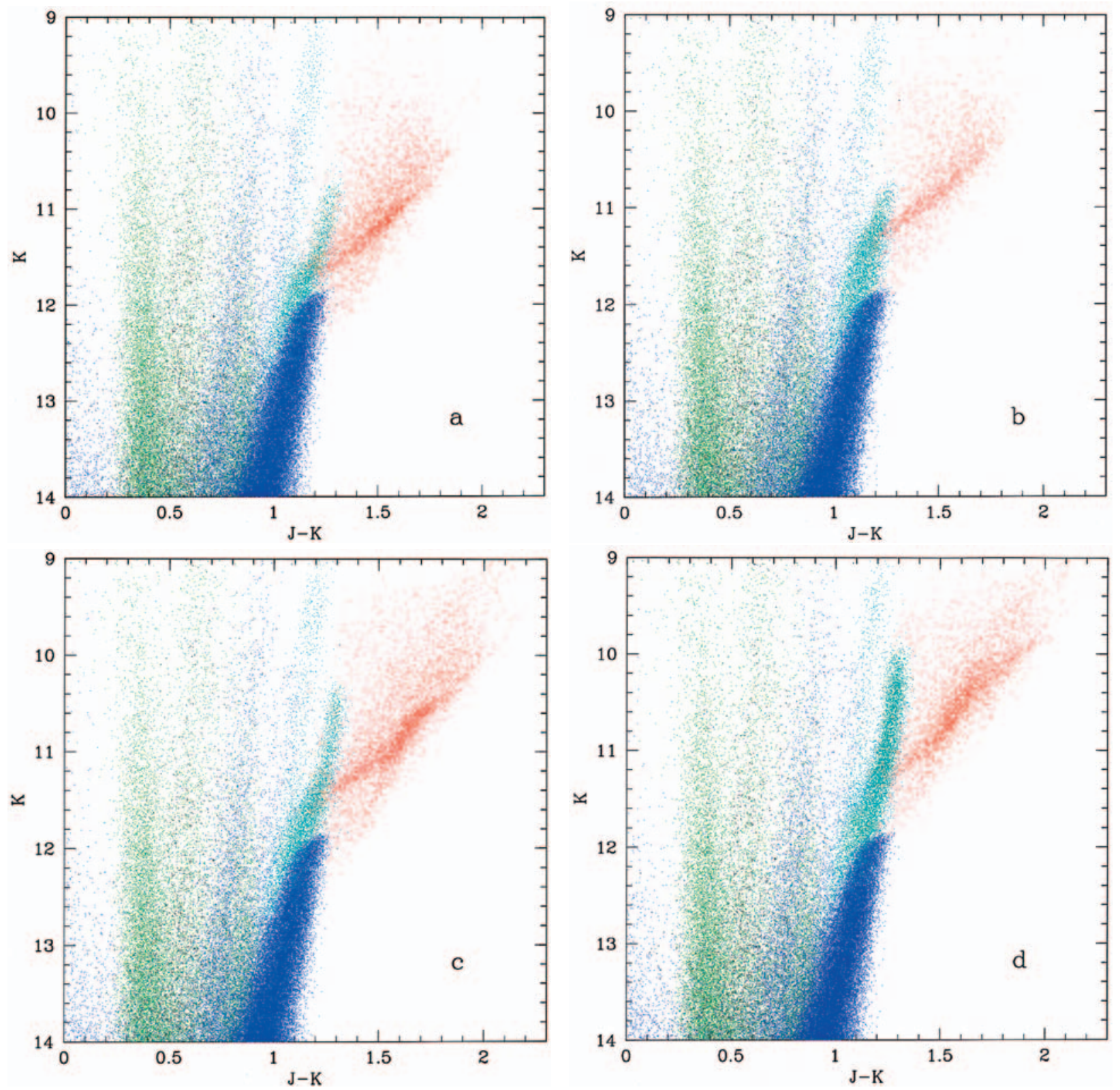

Fig. 11. Simulated H-R diagram based on TP-AGB tracks with variable molecular opacities (i.e. Marigo 2002). Note the departure of the C-star population away from the red giant branch towards redder colours. The mass loss prescriptions is taken from Vassiliadis \& Wood (1993), assuming that AGB stars are either fundamental-mode or first overtone pulsators. Panel a) case $\log T_{\mathrm{b}}^{\mathrm{dred}}=6.4$, fundamental mode. Panel b) case $\log T_{\mathrm{b}}^{\text {dred }}=6.45$, fundamental mode. Panel c) case $\log T_{\mathrm{b}}^{\mathrm{dred}}=6.4$, first overtone. Panel d) $\operatorname{case} \log T_{\mathrm{b}}^{\text {dred }}=6.45$, first overtone.

remarks in Sect. 4.2.1). Instead, for a larger $T_{\mathrm{b}}^{\mathrm{dred}}$, the consequent later onset of the third dredge-up is able to shift the entire red tail to the right luminosities, as shown in Fig. 11d.

From a comparison with our reference plot of Fig. 4, we can conclude that the simulation of Fig. $11 \mathrm{~d}$ best reproduces the observed morphology of the red tail. On the other hand, the introduction of the first overtone hypothesis produces an undesirable effect in the sequence of old O-rich stars: they now extend to much higher luminosities (up to $K=10.4$ and $K=10.0$ in Figs. 11c and d, respectively) than observed (up to $K=11.2$ in Fig. 4).

As a matter of fact, nowadays the observational scenario of long-period variables (LPVs) appears quite complex, mainly due to the observed mixture of different pulsation modes, and the lively controversy about their assignment (see e.g. Wood et al. 1999; Feast 1999; Whitelock \& Feast 2000; Cioni et al. 2001; Noda et al. 2002). The situation gets even more intricate if one considers the evidence that some LPVs are found to be multi-period pulsators (Bergeat et al. 2002), and the possibility of an evolutionary path across different period-luminosity relations, so that LPVs may switch pulsation modes while evolving along the AGB (Cioni et al. 2001).

Without entering the open debate on the dominant pulsation mode, we perform a very simple test on the basis of purely theoretical considerations. Considering that the red tail of C-stars is somewhat better reproduced with the firstovertone assumption which, instead, does not seem to suitably describe the O-rich branch, we assume a mixed population of 

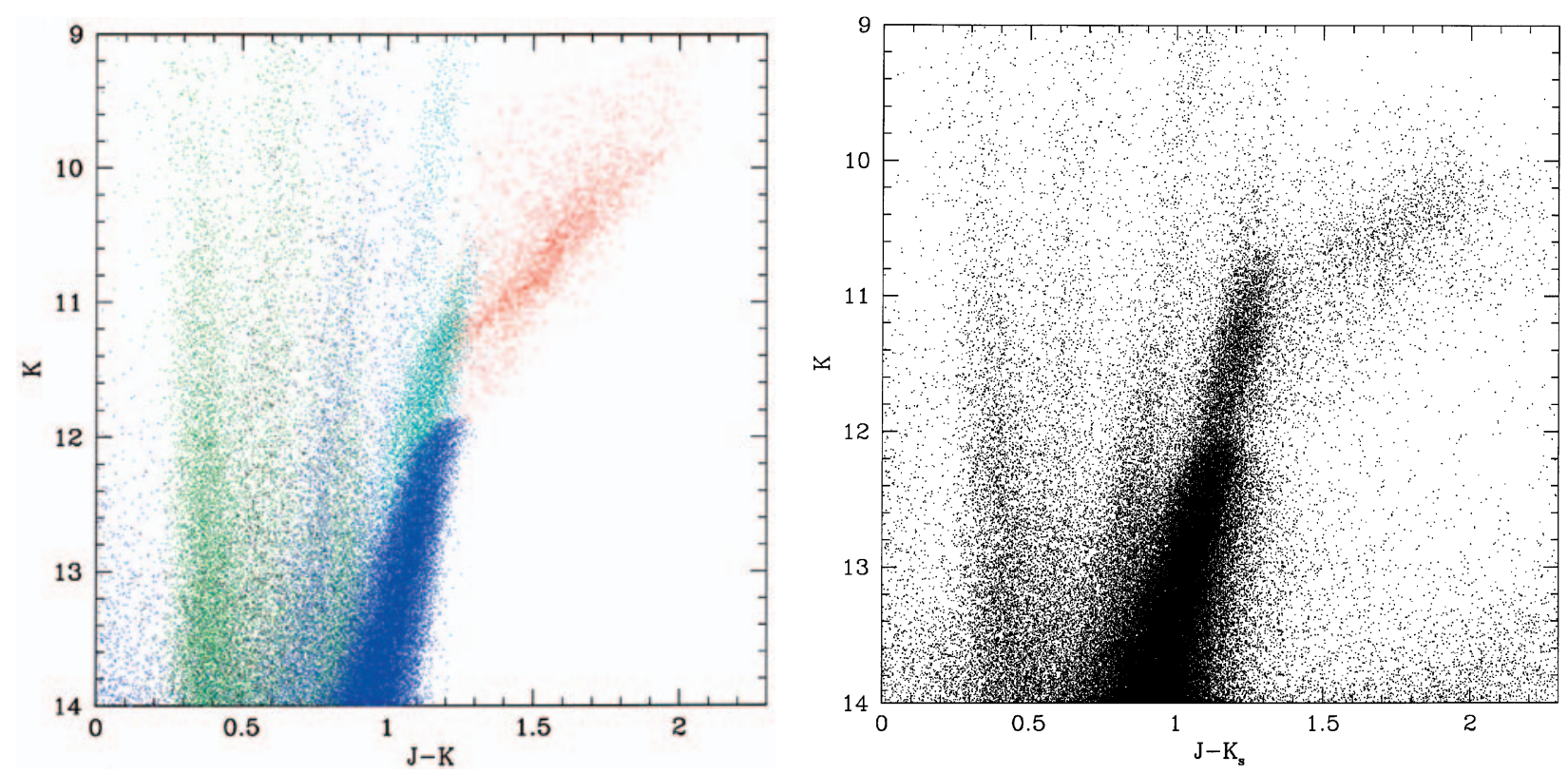

Fig. 12. Left panel: Simulated CMD based on TP-AGB tracks with variable molecular opacities (i.e. Marigo 2002) and log $T_{\mathrm{b}}^{\mathrm{dred}}=6.45$, assuming an half-and-half mixture of fundamental-mode and first-overtone pulsators for the stars which evolve into the C-star phase. Right panel: The same diagram for the selected 2MASS data. Notice the very good description of all CMD features, including the C-star red tail (in red) and the plume of O-rich low-mass TP-AGB stars (in cyan).

fundamental-mode and first-overtone pulsators, with the following composition:

1. All stars with mass lower than $1.3 M_{\odot}$, and larger than $3.5 M_{\odot}$, are fundamental-mode pulsators just because they do not evolve into a C-star phase;

2. The remaining stars are assigned either as fundamentalmode or as first-overtone pulsators. We assume a mix of 50 percent between the two types.

Assumption (2) may be not realistic enough, because the simulated stars do not switch between pulsation modes as they evolve, but they can just follow one between two different evolutionary paths. If this is an oversimplification of the problem - unavoidable because the mechanisms leading to change the pulsation mode cannot be presently modelled - at the same time it is a reasonable and instructive experiment. In Fig. 12 we present the resulting simulation, compared with the selected 2MASS data. We can notice, in fact, that the resulting sequence of O-rich giants is noticeably shortened in luminosity, as expected.

We may therefore conclude that now our final simulation actually meets 2MASS and DENIS observations, as all CMD features are well reproduced by the models, including the $C$ star red tail and the plume of $O$-rich low-mass TP-AGB stars.

\section{Concluding remarks}

In this paper, we have shown that most features seen in the 2MASS and DENIS $K_{\mathrm{s}}$ vs. $\left(J-K_{\mathrm{s}}\right)$ diagram are quite well described by our present stellar models. In particular, the observed tail towards the reddest $\left(J-K_{\mathrm{s}}\right)$ colours provides a direct and stringent probe to the evolutionary theory of TPAGB stars. In this regard, we have pointed out that:

1. The red tail of carbon stars is missed by models in which the TP-AGB evolution is followed using solar-scaled molecular opacities.

2. This failure can hardly be caused by some hypothetical inadequacy in the present-day $T_{\text {eff }}$ vs. $(J-K)$ relations for $\mathrm{C}$-stars. Our tests indicate that in order to reach the observed $(J-K)$ colours of C-stars with "solar-scaled opacity" models, quite high mean values of the $\mathrm{C} / \mathrm{O}$ ratio should be exhibited by the bulk of carbon stars, which seems unrealistical.

3. Instead, the red tail comes out naturally of TP-AGB models which are calculated with variable molecular opacities (cf. Marigo 2002), i.e. opacities consistently computed for the current chemical composition of the stellar envelope. This can be understood considering that, as the third dredge-up makes the $\mathrm{C} / \mathrm{O}$ ratio increase above 1 , drastic changes in the main sources of molecular opacities cause both the transition from $\mathrm{M}$ - to $\mathrm{C}$-spectral types, and a significant decrease of the effective temperature. The latter aspect has been so far largely ignored in AGB evolution models. Contrary to what claimed by Mouhcine \& Lançon (2002), the present study demonstrates that in order to model properly the properties of $\mathrm{AGB}$, in particular C-rich, stellar populations, one cannot ignore the large effects opacity have on the effective temperature of C-stars, and more generally on the AGB evolution.

4. Our simulations with "variable opacity" models provide a few additional useful indications about the TP-AGB evolution. For instance, models with $\log T_{\mathrm{b}}^{\text {dred }}=6.45$ seem to reproduce well the $K$-band luminosity of red tail C-stars. This means that the onset of the third dredge up should 
occur at slightly larger core masses, as compared to previous models by Marigo et al. (1999).

5. Moreover, in the context of the present models, based on the use of Vassiliadis \& Wood (1993) prescription for mass loss and an empirical $T_{\text {eff }}$ vs. $(J-K)$ relation, we may derive two indications about the pulsation modes of variable AGB stars. First, the colour extension of the red tail may imply a significant fraction of first-overtone pulsators among C-type stars. However, the same extension could be reproduced with fundamental-mode pulsators if circumstellar reddening starts to be important for C-stars with $(J-K)_{0} \sim 1.8$, a point that has still to be properly investigated. Second, the luminosity extension of the sequence populated by O-rich low-mass TP-AGB stars would suggest that most of M-type variables are fundamental-mode pulsators.

All these indications are being used to calibrate our TP-AGB models, from which extended sets of theoretical isochrones at varying metallicity will be derived and soon made available. Of course, new models will be computed for the variable-opacity case only.

Acknowledgements. We would like to thank our referee Dr. M. Bessell, and P. R. Wood, for useful remarks that improved the final version of the paper. We thank M. Groenewegen for his fine calibration of the parameters used in the Galaxy model and for useful comments. This publication makes use of data products from the Two Micron All Sky Survey, which is a joint project of the University of Massachusetts and the Infrared Processing and Analysis Center/California Institute of Technology, funded by the National Aeronautics and Space Administration and the National Science Foundation. The DENIS data is the result of an extended international collaboration with the use of ESO telescopes. We acknowledge financial support from the MIUR-COFIN 2001 project "The tridimensional structure of the Galaxy" (number 2001028112).

\section{References}

Alexander, D. R., \& Ferguson, J. W. 1994, ApJ, 437, 879

Bergbusch, P. A., \& VandenBerg, D. A. 2001, ApJ, 566, 322

Bergeat, J., Knapik, A., \& Rutily, B. 2001, A\&A, 369, 178

Bergeat, J., Knapik, A., \& Rutily, B. 2002, A\&A, 390, 987

Bertelli, G., Bressan, A., Chiosi, C., Fagotto, F., \& Nasi, E. 1994, A\&AS, 106, 275

Bessell, M. S., Wood, P. R., \& Lloyd Evans, T. 1981, MNRAS, 202, 59

Bessell, M. S., \& Brett, J. M. 1988, PASP, 100, 1134

Chabrier, G. 2001, ApJ, 554, 1274

Chieffi, A., Domínguez, I., Limongi, M., et al. 2001, ApJ, 554, 1159

Cioni, M. R., Habing, H. J., Loup, C., et al. 1999, in New Views of the Magellanic Clouds, ed. Y.-H. Chu, N. Suntzeff, J. Hesser, \& D. Bohlender, IAU Symp., 190, 385

Cioni, M. R., Loup, C., Habing, H. J., et al. 2000, A\&AS, 144, 235

Cioni, M.-R. L., Marquette, J.-B., Loup, C., et al. 2001, A\&A, 377, 945

Cohen, J. G., Frogel, J. A., Persson, S. E., et al. 1981, ApJ, 249, 481

Cutri, R. M., Skrutskie, M. F., van Dyk, S., et al. 2002, Explanatory Supplement to the 2MASS Second Incremental Data Release http://www.ipac. caltech.edu/2mass/releases/second/ doc/explsup.html
Domínguez, I., Chieffi, A., Limongi, M., et al. 1999, ApJ, 524, 226

Epchtein, N., de Batz, B., Capoani, L., et al. 1997, The Messenger, 87, 27

Feast, M. 1999, in Asymptotic Giant Branch Stars, ed. T. Le Bertre, A. Lebre, \& C. Waelkens, IAU Symp., 191, 109

Fluks, M. A., Plez, B., Thé, P. S., et al. 1994, A\&AS, 105, 311

Forestini, M., \& Charbonnel, C. 1997, A\&AS, 123, 241

Frogel, J. A., Mould, J., \& Blanco, V. M. 1990, ApJ, 352, 96

Gilmore, G. 1984, MNRAS, 207, 223

Girardi, L., Bressan, A., Bertelli, G., \& Chiosi, C. 2000, A\&AS, 141, 371

Girardi, L., \& Salaris, M. 2001, MNRAS, 323, 109

Girardi, L., Bertelli, G., Bressan, A., et al. 2002, A\&A, 391, 195

Girardi, L., \& Marigo, P. 2003, in CNO in the Universe, ed. C. Charbonnel, D. Schaerer, \& G. Meynet, ASP Conf. Ser., in press [astro-ph/0302020]

Groenewegen, M. A. T., Girardi, L., Hatziminaoglou, E., et al. 2002, A\&A, 392, 741

Herwig, F. 2000, A\&A, 360, 952

Herwig, F., Blöcker, T., Schönberner, D., et al. 1997, A\&A, 324, L81

Hughes, S. M. G., \& Wood, P. R. 1990, AJ, 99, 784

Iglesias, C. A., \& Rogers, F. J. 1993, ApJ, 412, 752

Karakas, A. I., Lattanzio, J. C., \& Pols, O. R. 2002, PASA, 19, 515

Lejeune, T., \& Schaerer, D. 2001, A\&A, 366, L538

Loidl, R., Lançon, A., \& Jørgensen, U. G. 2001, A\&A, 371, 1065

Marigo, P. 1998, A\&A, 340, 463

Marigo, P. 2002, A\&A, 387, 507

Marigo, P., Bressan, A., \& Chiosi, C. 1996, A\&A, 313, 545

Marigo, P., Bressan, A., \& Chiosi, C. 1998, A\&A, 331, 564

Marigo, P., Girardi, L., \& Bressan, A. 1999, A\&A, 344, 123

Marigo, P., \& Girardi, L. 2001, A\&A, 377, 132

Mouhcine, M., \& Lançon, A. 2002, A\&A, 393, 149

Ng, Y. K., Bertelli, G., Chiosi, C., \& Bressan, A. 1997, A\&A, 324, 65

Nikolaev, S., \& Weinberg, M. D. 2000, ApJ, 542, 804

Noda, S., Takeuti, M., Abe, F., et al. 2002, MNRAS, 330, 137

Osmer, P. S., Kennefick, J. D., Hall, P. B., \& Green, R. F. 1998, ApJS, 119,189

Pagel, B. E. J., \& Tautvaisiene, G. 1998, MNRAS, 299, 535

Prandoni, I., Wichmann, R., da Costa, L., et al. 1999, A\&A, 345, 448

Richer, H. B., Olander, N., \& Westerlund, B. E. 1979, ApJ, 230, 724

Rocha-Pinto, H. J., Maciel, W. J., Scalo, J., \& Flynn, C. 2000, A\&A, 358,850

Skrutskie, M. F., Schneider, S. E., Stiening, R., et al. 1997, in The Impact of Large Scale Near-IR Surveys, ed. F. Garzon (Dordrecht: Kluwer), 25

Staveley-Smith, L., Kim, S., Calabretta, M. R., Haynes, R. F., \& Kesteven, M. J. 2003, MNRAS, 339, 87

Straniero, O., Chieffi, A., Limongi, M., et al. 1997, ApJ, 478, 332

van der Marel, R. P., \& Cioni, M.-R. 2001, AJ, 122, 1807

van der Marel, R. P., Alves, D. R., Hardy, E., \& Suntzeff, N. B. 2002, AJ, 124, 2639

van Loon, J. 1999, Ph.D. Thesis, Universiteit van Amsterdam

Ventura, P., D’Antona, F., \& Mazzitelli, I. 1999, ApJ, 524, L111

Vassiliadis, E., \& Wood, P. R. 1993, ApJ, 413, 641

Weinberg, M. D., \& Nikolaev, S. 2001, ApJ, 548, 712

Weiss, A., \& Salaris, M. 1999, A\&A, 346, 897

Whitelock, P., \& Feast, M. 2000, MNRAS, 319, 759

Wood, P. R., Alcock, C., Allsman, R. A., et al. 1999, in Asymptotic Giant Branch Stars, ed. T. Le Bertre, A. Lebre, \& C. Waelkens, IAU Symp., 191, 151 